12,13

\title{
K вопросу о влиянии неоднородного диэлектрического покрытия на характеристики металлической поверхности
}

\author{
(C) В.В. Погосов \\ Национальный университет „Запорожская политехника“, \\ Запорожье, Украина \\ E-mail: vpogosov@zntu.edu.ua
}

Поступила в Редакцию 9 августа 2021 г.

В окончательной редакции 6 сентября 2021 г.

Прнинята к публикации 11 сентября 2021 г.

В рамках квантовостатистичекого функционала и метода Ритца рассмотрена задача по нахождению удельной поверхностной энергии и работы выхода электронов металлической плоской поверхности с неоднородным диэлектрическим покрытием, учтенным в приближении сплошной среды. Для однородного покрытия расчетные величины нечувствительны к выбору однопараметрических функций для электронного профиля, но чувствительны к градиентному ряду кинетической энергии невзаимодействующих электронов. Вычисления выполнены для Al, Na и проведено сравнение с расчетами методом Кона-Шема. Аналитически установлена связь теории метода Ритца для неоднородных покрытий с расчетами методом Кона-Шема [ФТT 55, 177 (2013)] поверхностной энергии и работы выхода электронов для металл-диэлектрических наносандвичей. Предлагается влияние неоднородности покрытия оценивать масштабированием характеристик металлической поверхности с однородным покрытием. Обсуждается возможность применения полученных результатов в различных экспериментальных ситуациях.

Ключевые слова: работа выхода, барьер Шоттки, диэлектрическое покрытие.

DOI: 10.21883/FTT.2022.01.51841.187

\section{1. Введение}

Исследования электронных свойств металлической поверхности со сложным покрытием представляют интерес для современных технологий с целью создания, например, материалов, обладающих значительным сенсорным откликом и селективностью. Конечная задача индустрии покрытий в конструировании „умных“ покрытий, которые сохраняли свои свойства в непредсказуемых условиях.

О сложности получения подходящих для исследования объектов и методов измерения говорит сравнительно малое количество экспериментальных исследований, целью которых, как правило, являются квантово-размерные эффекты. Одной из важнейших равновесных характеристик металлических наноструктур является работа выхода электронов.

Технологический интерес представляют пленки (пластины), выращенные на различных подложках [1-3]. Например, в работе [3] экспериментально исследовались пленки $\mathrm{Pb}(111)$ толщиной $L$ от 1 до 16 монослоев, выращенные на подложке $\mathrm{Ge}(111)$. Измерялась работа выхода в вакуум методом фотоэмиссионной спектроскопии.

В приближении сплошной среды, при расчетах металл-диэлектрических интерфейсов, изолятор характеризуется лишь диэлектрической постоянной $\varepsilon$. В рамках такого подхода, используя приближения локальной плотности (LDA), выполнены многочисленные вычисления поляризуемости и поверхностного плазмонно- го резонанса в металлических наносферах и нитях в различных диэлектрических матрицах, нанопленок на диэлектрических подложках (см., например, [4]), а также предсказано изменение знака работы выхода позитронов в зависимости от $\varepsilon$ диэлектрического покрытия металла [5]. В рамках метода Кона-Шема нами рассчитывались поверхностные характеристики металл-диэлектрических наносандвичей $[6,7]$.

Высота барьера Шоттки $\Phi$ контакта металл-изолятор оценивается на основе правила Шоттки-Мотта $[8,9]$ с характеристиками изолированных друг от друга металла и изолятора

$$
\Phi \approx W-\chi,
$$

где $W$ и $-\chi-$ работа выхода электронов из металла в вакуум и глубина залегания зоны проводимости электронов в изоляторе до контакта. При малых зазорах металла и изолятора хвост электронного распределения металла находится под влиянием поляризации изолятора. Для уточнения правила (1) можно ввести зависимость $W(\varepsilon)$, где $\varepsilon-$ диэлектрическая константа изолятора. Тогда, исходя из анализа, можно полагать, что авторами [3] наблюдался пиннинг уровня Ферми в пленке $\mathrm{Pb}(111)$ : все значения $W(\varepsilon, L)$ находятся ниже примерно $4 \mathrm{eV}$ (это значение соответствует $\chi$ для $\mathrm{Ge}$ ), а размерные флуктуации $\Delta W(\varepsilon, L) \in(0.2,0.5) \mathrm{eV}$. Примерно такие значения $\Delta W(L)$ получены нами [7] для Vacuum/ $/ \mathrm{Al} / \mathrm{Al}_{2} \mathrm{O}_{3}$ в отсутствии пиннинга (экспериментальные значения работы выхода $\mathrm{Al}$ и $\mathrm{Pb}$ близки друг другу).

Проблема описания металла с неоднородным покрытием непосредственно связана с вопросом о анизотро- 
пии работы выхода или о локальной работе выхода, которая по сути соответствует локальному значению эффективного одноэлектронного потенциала, зависящего от координат [10-15]. Исследования подобного рода принято выполнять либо методом Кона-Шема [12,14], либо $a b$ initio методами $[11,13,15]$, которые зачастую не позволяют провести детальный анализ получаемых результатов. Такой анализ возможен в рамках вариационного метода Ритца с использованием простейших пробных функций, моделирующих профиль электронной плотности.

Целью работы является определение методом Ритца работы выхода и поверхностной энергии плоской металлической поверхности, участки которой покрыты различными диэлектриками.

\section{2. Постановка задачи}

Соответственно цели работы, на первый взгляд, технически удобно использовать цилиндрическую систему координат. Представим, например, что на торце макроскопического цилиндра часть его площади покрыта диэлектриком, а остальная поверхность цилиндра покрыта другим диэлектриком. Предельный переход к бесконечному радиусу цилиндра усложняется возникающими неопределенностями, в частности, электростатической части задачи. Это вынуждает использовать сферическую систему координат.

Рассмотрим макроскопическую металлическую сплошную сферу радиуса $R$, покрытую слоем диэлектриков с различными постоянными $\varepsilon_{i}$ (приближение сплошной среды).

Руководствуясь опытом нашей предыдущей работы [14], для простоты вычислений мы приняли толщины диэлектриков бесконечно большими. Достаточно быстрое убывание электронного распределения вне металла (примерно на расстояния 5-10 ангстрем) позволяет пренебречь эффектом толщины этого покрытия, минимальная толщина которого должна быть гораздо больше моноатомного (или мономолекулярного) слоя диэлектрика. Длина свободного пробега электронов в диэлектриках - это десятки ангстрем [16].

Для аналитического решения задачи положим, что на металлической поверхности граничат всего два диэлектрика 1 и $2(i=1,2)$. Область диэлектрика с $\varepsilon_{1}$ может быть задана, например, полярным углом $\theta_{m}$ (рис. 1). Рис. 1 в случае $\varepsilon_{2}=1$ может быть применен к металлической капле, лежащей на диэлектрической подложке с $\varepsilon_{1}$.

Задавая долю

$$
\alpha=S_{1} / S
$$

поверхности сферы, занятую диэлектриком $1\left(S_{1}=2 \pi R h\right.$, $h$ - высота сферического сегмента), получаем значение пограничного угла

$$
\cos \theta_{m}=1-h / R=1-2 \alpha .
$$

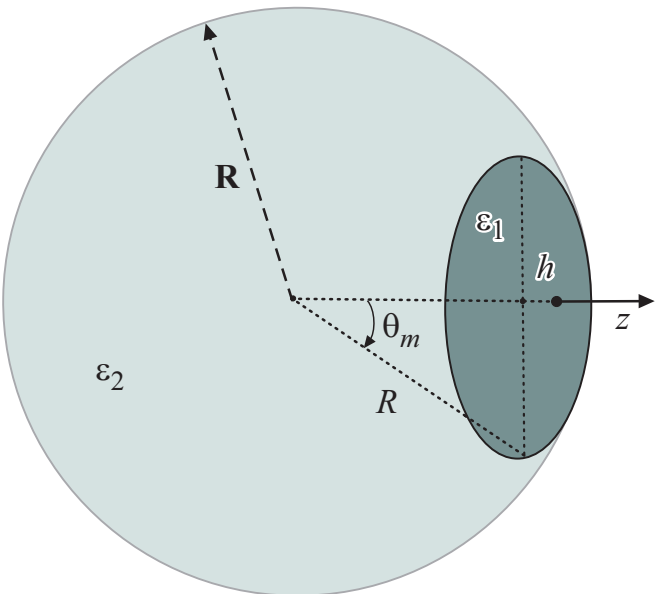

Рис. 1. Геометрическая схема металлической поверхности, покрытой двумя разными диэлектриками.

В модели желе металла распределение положительного (ионного) заряда является однородным и изотропным, и задается функцией Хевисайда

$$
\rho(r)=\bar{\rho} \Theta(r-R), \quad \bar{\rho}=\bar{n}
$$

(оно одинаково для областей 1 и 2$), \Theta(r-R)=$ $=\{1, r \leq R ; 0, r>R\}$. Здесь $\bar{n}=\left(4 \pi r_{s}^{3} / 3\right)^{-1}-$ концентрация электронного газа в объеме металла, $r_{s}-$ среднее расстояние между электронами.

В изотропном случае, на примере желе-сферы в вакууме $\left(\varepsilon_{i}=1\right)$, ограничиваясь электронными профилями $n(r)$ в виде однопараметрических пробных функций (метод Ритца), известно, что эффективный радиус электронного облака $R^{\prime}$ отличается от $R$ [17]. Это отличие $\propto R^{-1}$ и находится из условия электронейтральности

$$
\int d \mathbf{r} v(\mathbf{r})=0, \quad v(\mathbf{r}) \equiv n(\mathbf{r})-\rho(\mathbf{r})
$$

Действуя в иной манере, для желе-сферы в вакууме (то же самое можно утверждать и для шара с однородным диэлектрическим покрытием), радиус электронного облака $R^{\prime}$ приравняем к радиусу ионного желе $R$, а профиль выберем в виде

$$
\begin{gathered}
n(r)=\bar{n} \begin{cases}1-A e^{(r-R) / \lambda}, & r \leq R, \\
B e^{(R-r) / \lambda}, & r>R,\end{cases} \\
A=\frac{1}{2} f, \quad B=1-A,
\end{gathered}
$$

где $f$ учитывает отличие $R^{\prime}$ от $R$.

В результате интегрирования в (5) получаем

$$
f=1+\frac{2}{\beta}-\frac{4}{\beta^{3}},
$$

где $\beta=\lambda / R \ll 1$. 
В плоском случае $(R \rightarrow \infty, z=r-R)$ коэффициенты $A=B=1 / 2$ соответствуют профилю, предложенному в [18] при обсуждении работы выхода электронов для разных кристаллографических граней металла и впервые использованному в методе функционала плотности для плоской металлической поверхности в [19].

В соответствии с рис. 1, каждой области соответствуют свои профили. В качестве первого шага используем (6) и для шара с неоднородным покрытием, вводя соответствующие индексы для $n(r), A, B, f$

$$
n_{i}(\mathbf{r})= \begin{cases}n_{1}(r), & \theta \in\left(0, \theta_{m}\right), \\ n_{2}(r), & \theta \in\left(\theta_{m}, \pi\right),\end{cases}
$$

Известно, что для металлов с однородным покрытием работа выхода электронов $W(\varepsilon)$ уменьшается с увеличением $\varepsilon$ [14]. Нанесение на поверхность металла разных диэлектриков $\left(\varepsilon_{1} \neq \varepsilon_{2}\right)$ приведет к перетеканию электронной жидкости вблизи поверхности из области 1 в область 2 (или наоборот) вследствие возникающей контактной разности потенциалов. Распределения $n_{1}(\mathbf{r})$ или $n_{2}(\mathbf{r})$ при этом окажутся сдвинутыми в противоположные стороны по нормали вглубь либо металла, либо диэлектрика. Такой эффект можно отразить введением параметра $\delta$ в соответствующие коэффициенты (7):

$$
A_{i}=\frac{1}{2} f_{i}+\delta_{i}, \quad B_{i}=1-A_{i} .
$$

Параметры $A_{1,2}, B_{1,2}$ и $\lambda_{1,2}$ характеризуют размеры локальных дипольных барьеров областей 1 и 2 .

Интегрирование в (5), с учетом (6)-(9) и (3) приводит к соотношению

$$
\delta_{1}=-\left(\frac{\alpha}{1-\alpha}\right) \frac{\lambda_{2}}{\lambda_{1}} \delta_{2}+O\left(\frac{1}{\beta_{1}^{2}}, \frac{1}{\beta_{2}^{2}}\right) .
$$

Интересно отметить, что в плоском случае $(R \rightarrow \infty, z=r-R)$ параметры $\delta_{i} \quad$ должны давать неисчезающий вклад в (5), что связано с фиксированным значением коэффициента $\alpha$ независимо от перехода $R \rightarrow \infty$. Противоположный знак $\delta_{1}$ и $\delta_{2}$ означает потерю локальной электронейтральности в областях 1 и 2 вследствие перетекания электронов, но при сохранении полной электронейтральности сферы. В случае $\alpha=0,1$ имеем $\lambda_{1}=\lambda_{2}$ и $\delta=0$ (однородное покрытие), а в случае $\alpha=1 / 2$, когда половины площади сферы покрыты разными диэлектриками, $\delta_{1}=-\left(\lambda_{2} / \lambda_{1}\right) \delta_{2}$.

Используя (4) в плоском случае и подсчитывая число электронов, перетекающих из одной области в другую, можно составить равенство

$$
\bar{\rho}\left[(1-\alpha) S \delta_{1}\right]=-\bar{\rho}\left(\alpha S \delta_{2}\right),
$$

из которого следует

$$
\delta_{1}=-\left(\frac{\alpha}{1-\alpha}\right) \delta_{2}
$$

Тогда, из сопоставления (10) и (11) для выбранного класса пробных функций, получим важное условие

$$
\lambda_{1}=\lambda_{2}
$$

Рассмотрим вначале случай $\alpha=0,1$ на рис. 1 в пределе $R \rightarrow \infty-$ плоскую поверхность металла, покрытую однородным диэлектриком.

\section{3. Однородное покрытие плоской поверхности}

В модели обычного желе (J) и методе Ритца используем однопараметрический профиль электронов $n(z)$ и однородное распределение положительно заряженного фона $\rho(z)=\bar{n} \Theta(-z)$. Квантовостатистический функционал с объемной плотностью энергии электронов $g$ в LDA, состоит из: квазиоднородной кинетической энергии невзаимодействующего электронного газа (здесь и дальше атомные единицы Хартри)

$$
g_{\mathrm{t}}(n)=n \frac{3}{10}\left(3 \pi^{2} n\right)^{2 / 3} ;
$$

членов градиентного разложения Вейцзеккера-Киржница-Ходжа [20] кинетической энергии, содержащего четные степени градиентов

$$
\begin{aligned}
g_{\mathrm{g}} & \equiv g_{\mathrm{g} 1}+g_{\mathrm{g} 2}+g_{\mathrm{g} 3}+g_{\mathrm{g} 4}=\frac{|\nabla n|^{2}}{72 n}+\frac{n^{1 / 3}}{540\left(3 \pi^{2}\right)^{2 / 3}} \\
& \times\left[\left(\frac{\nabla^{2} n}{n}\right)^{2}-\frac{9}{8}\left(\frac{\nabla^{2} n}{n}\right)\left|\frac{\nabla n}{n}\right|^{2}+\frac{1}{3}\left|\frac{\nabla n}{n}\right|^{4}\right]
\end{aligned}
$$

обменной энергии Дирака

$$
g_{\text {ex }}(n)=-n \frac{3}{4 \pi}\left(3 \pi^{2} n\right)^{1 / 3}
$$

и корреляционной энергии Пайнса-Нозьера

$$
g_{\text {cor }}(n)=n\left[0.0474+0.0155 \ln \left(3 \pi^{2} n\right)^{1 / 3}\right] .
$$

Нелокальная (электростатическая) составляющая энергии имеет вид

$$
E_{\mathrm{q}}(n)=\frac{1}{2} \int d \mathbf{r} \phi v
$$

[величина $v$ определена в (5)].

Электростатический потенциал $\phi$ находится решением уравнения Пуассона

$$
\nabla^{2} \phi(z)=-\frac{4 \pi}{\epsilon(z)} v(z)
$$

с граничным условием $\phi(z) \rightarrow 0$ при $z \rightarrow+\infty$. Функция $\epsilon(z)$ равна 1 внутри металла, где электроны и ионы находятся в вакууме, и равна $\varepsilon$ снаружи металла. Решение уравнения (15) имеет вид

$$
\phi(z)=\bar{\phi}-4 \pi \int_{-\infty}^{z} d z^{\prime}\left(z-z^{\prime}\right) \frac{v\left(z^{\prime}\right)}{\epsilon\left(z^{\prime}\right)}
$$


Таблица 1. Значения коэффициентов в выражении (26), рассчитанные с использованием функций (21) (верхнее значение) и (22) (нижнее значение)

\begin{tabular}{c|c|c|c|c|c|c|c|c}
\hline$C_{\mathrm{q}}$ & $C_{\mathrm{t}}$ & $C_{\mathrm{ex}}$ & $C_{\text {cor }} \cdot 10^{3}$ & $C_{\mathrm{g} 1} \cdot 10^{3}$ & $C_{\mathrm{g} 2} \cdot 10^{4}$ & $C_{\mathrm{g} 3} \cdot 10^{4}$ & $C_{\mathrm{g} 4} \cdot 10^{4}$ & $C_{\mathrm{Ws}}$ \\
\hline 0.785 & -1.842 & 0.2502 & 6.589 & 9.627 & 5.115 & -4.832 & 1.611 & $-1 / 2$ \\
1.886 & -2.179 & 0.3288 & 3.499 & 6.944 & 2.861 & -3.359 & 1.120 & -0.693
\end{tabular}

со значением потенциала в глубине металла

$$
\bar{\phi} \equiv \phi(-\infty)=-4 \pi \int_{-\infty}^{+\infty} d z z \frac{v(z)}{\epsilon(z)}<0
$$

Удельная поверхностная энергия в модели обычного желе равна по определению

$$
\sigma_{\mathrm{J}}=\int_{-\infty}^{\infty} d z\left[g(n(z))+\frac{1}{2} \phi(z) v(z)-g(\bar{n}) \Theta(-z)\right]
$$

а в модели стабильного желе $[21,22]$

$$
\sigma=\sigma_{\mathrm{J}}+\langle\delta v\rangle_{\mathrm{WS}} \int_{-\infty}^{0} d z v(z)
$$

Стабилизационный потенциал имеет вид

$$
\langle\delta v\rangle_{\mathrm{WS}}=2\left(0.0026+k_{\mathrm{F}} / 8 \pi-k_{\mathrm{F}}^{2} / 10\right),
$$

$k_{\mathrm{F}}=\left(3 \pi^{2} \bar{n}\right)^{2 / 3}$.

Работа выхода электронов в модели стабильного желе определяется как

$$
W=-\bar{\phi}-\frac{d g(\bar{n})}{d \bar{n}}-\langle\delta v\rangle_{\mathrm{WS}} .
$$

Для сравнительного анализа вычисления выполнены для двух функций: „антисимметричной“ функции

$$
n(z)=\bar{n} \begin{cases}1-\frac{1}{2} e^{z / \lambda}, & z \leq 0 \\ \frac{1}{2} e^{-z / \lambda}, & z \geq 0\end{cases}
$$

которая совпадает с (6) при $R \rightarrow \infty$, а также функции фермиевского вида

$$
n(z)=\frac{\bar{n}}{1+e^{z / \lambda}} .
$$

Подстановкой (21) и (22) в (16) и (17), а затем последующим интегрированием получаем

$$
\phi(z)=-2 \pi \bar{n} \lambda^{2} \begin{cases}1+\frac{1}{\varepsilon}-e^{z / \lambda}, & z \leq 0, \\ \frac{1}{\varepsilon} e^{-z / \lambda}, & z \geq 0,\end{cases}
$$

и

$$
\begin{aligned}
& \phi(z)= \\
& =\bar{n} \lambda^{2} \begin{cases}-\frac{1}{3} \pi^{3}\left(1+\frac{1}{\varepsilon}\right)-4 \pi \sum_{k=1}^{\infty}(-1)^{k} \frac{1}{k^{2}} e^{k z / \lambda}, & z \leq 0, \\
\frac{4 \pi}{\varepsilon} \sum_{m=1}^{\infty}(-1)^{m} \frac{1}{m^{2}} e^{-m z / \lambda}, & z \geq 0,\end{cases}
\end{aligned}
$$

соответственно (при $z=0$ потенциалы сшиваются).

Выражение (23) совпадает с соответствующим результатом работы [23]. В (23) и (24) при $z=0$ легко прослеживается связь со значением электростатического потенциала на границе проводящего шара в диэлектрике [24]. Для этого в сферическом случае нужно рассмотреть вклад ионного желе и электронного облака по-отдельности.

При выводе (24) во внутреннем интегрировании в (16) использовано разложение (22) по степеням малости $e^{-|z| / \lambda} \ll 1$. Такое разложение хорошо работает за исключением окрестности $z=0$. Интегрированием эта особенность нивелируется при выводе $\phi(z)$ и нахождении электростатической компоненты поверхностной энергии

$$
\sigma_{\mathrm{q}}=\frac{1}{8 \pi} \int_{-\infty}^{+\infty} d z(\nabla \phi)^{2} .
$$

Результат аналитических вычислений компактно может быть записан в виде

$$
\begin{aligned}
\sigma= & \bar{n}^{2} \lambda^{3} C_{\mathrm{q}}\left(1+\frac{1}{\varepsilon}\right)+\lambda\left(\bar{n}^{5 / 3} C_{\mathrm{t}}+\bar{n}^{4 / 3} C_{\mathrm{ex}}+\bar{n} C_{\mathrm{cor}}\right) \\
& +\frac{\bar{n}}{\lambda} C_{\mathrm{g} 1}+\frac{\bar{n}^{1 / 3}}{\lambda^{3}}\left(C_{\mathrm{g} 2}+C_{\mathrm{g} 3}+C_{\mathrm{g} 4}\right)+\bar{n} \lambda C_{\mathrm{WS}}\langle\delta v\rangle_{\mathrm{WS}} .
\end{aligned}
$$

Значения коэффициентов $C$ зависят от вида пробной функции и приведены в табл. 1.

На рис. 2 представлены для $\mathrm{Al}$ оптимальные значения вариационного параметра $\lambda(\varepsilon)$, характеризующего поверхностный профиль электронного распределения, найденные из условия

$$
\frac{d}{d \lambda} \sigma(\bar{n}, \varepsilon, \lambda)=0 .
$$

Значения $\lambda(\varepsilon)$ чувствительны, как к используемому градиентному ряду, так и к виду пробной функции. 


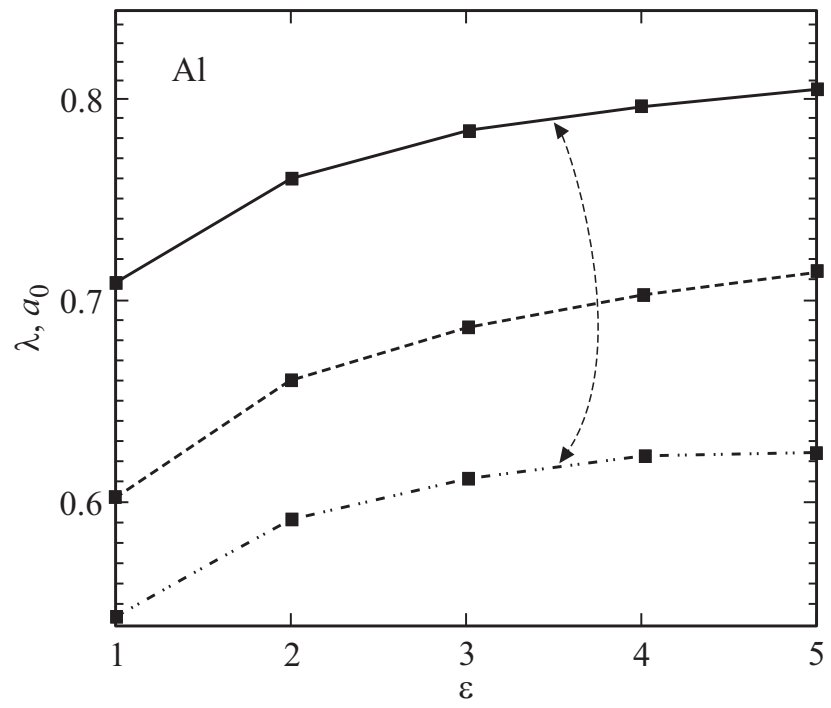

Рис. 2. Зависимость оптимизированного параметра $\lambda$ („ширины“ переходного слоя) в методе Ритца для поликристаллического $\mathrm{Al}\left(r_{s}=2.07 a_{0}\right)$ от диэлектрической постоянной однородного покрытия: для функции (21) и полного градиентного ряда (сплошная линия), с поправкой Киржница (штрихпунктирная линия); для функции (22) и полного градиентного ряда (пунктирная линия). $a_{0}-$ боровский радиус.

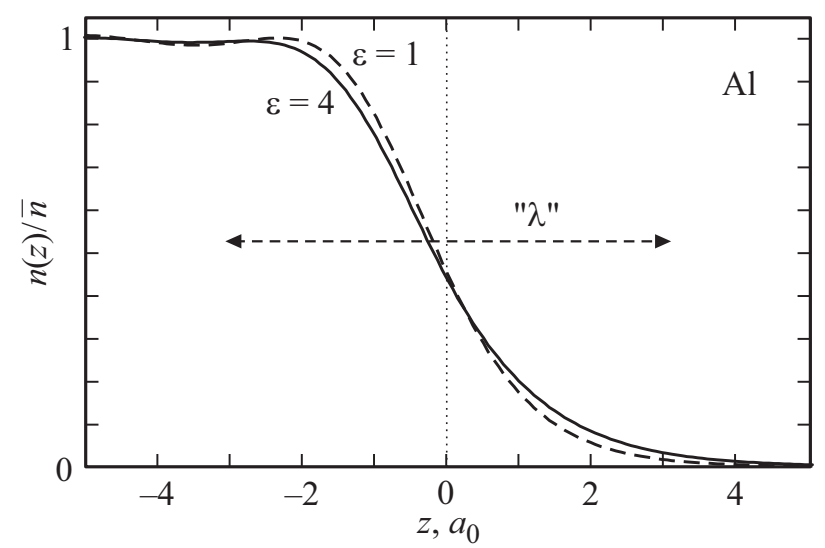

Рис. 3. Одномерные электронные профили, рассчитанные методом Кона-Шема.

С увеличением константы $\varepsilon$ покрытия величина параметра $\lambda$ растет, выходя на плато. Зависимость $\lambda(\varepsilon)$ качественно согласуется с поведением электронных профилей, рассчитанных методом Кона-Шема, но значения $\lambda$ значительно меньше, если условно провести сравнение с рис. 3, на котором видны фриделевские осцилляции электронной плотности.

Полностью самосогласованные вычисления в методе Кона-Шема и LDA приводят к более значительным величинам ширины поверхностного слоя, а сшивка с потенциалом изображения - к еще большим величинам [7]. Поэтому используемый метод Ритца частичного самосогласования процедуры, хотя и позволяет аналитическое рассмотрение подобных задач, проигрывает в воспроизведении профиля эффективного одноэлектронного потенциала $v_{\text {eff }}(z)$ вблизи поверхности при полном самосогласовании.

Полученную нами зависимость $\lambda(\varepsilon)$ можно прокомментировать и с точки зрения „механического“ равновесия металла с покрытием в терминах тензора натяжений или давлений [25].

Тензор натяжений содержит неэлектростатические составляющие, а также максвелловский тензор натяжений. В общем случае он зависит от константы покрытия $\varepsilon$ и дает вклад $\mp \frac{1}{8 \pi}(\nabla \phi)^{2}$ в нормальную и тангенциальную компоненту давления, соответственно, определяя тем самым зависимость профиля электронов $n(\varepsilon, z)$, т.е. $\lambda(\varepsilon)$, а также электростатическую составляющую поверхностной энергии (25). Условия равновесия поверхности требуют равенства нулю нормальной компоненты давления при отсутствии внешнего давления.

В методе Кона-Шема (см. рис. 3, а также рис. 3 в [14]) этот тензор натяжений отвечает за „вытягивание“ профилей $n(\varepsilon, z)$ и одноэлектронного эффективного потенциала $v_{\text {eff }}(z)$ в область диэлектрика, в тоже время к
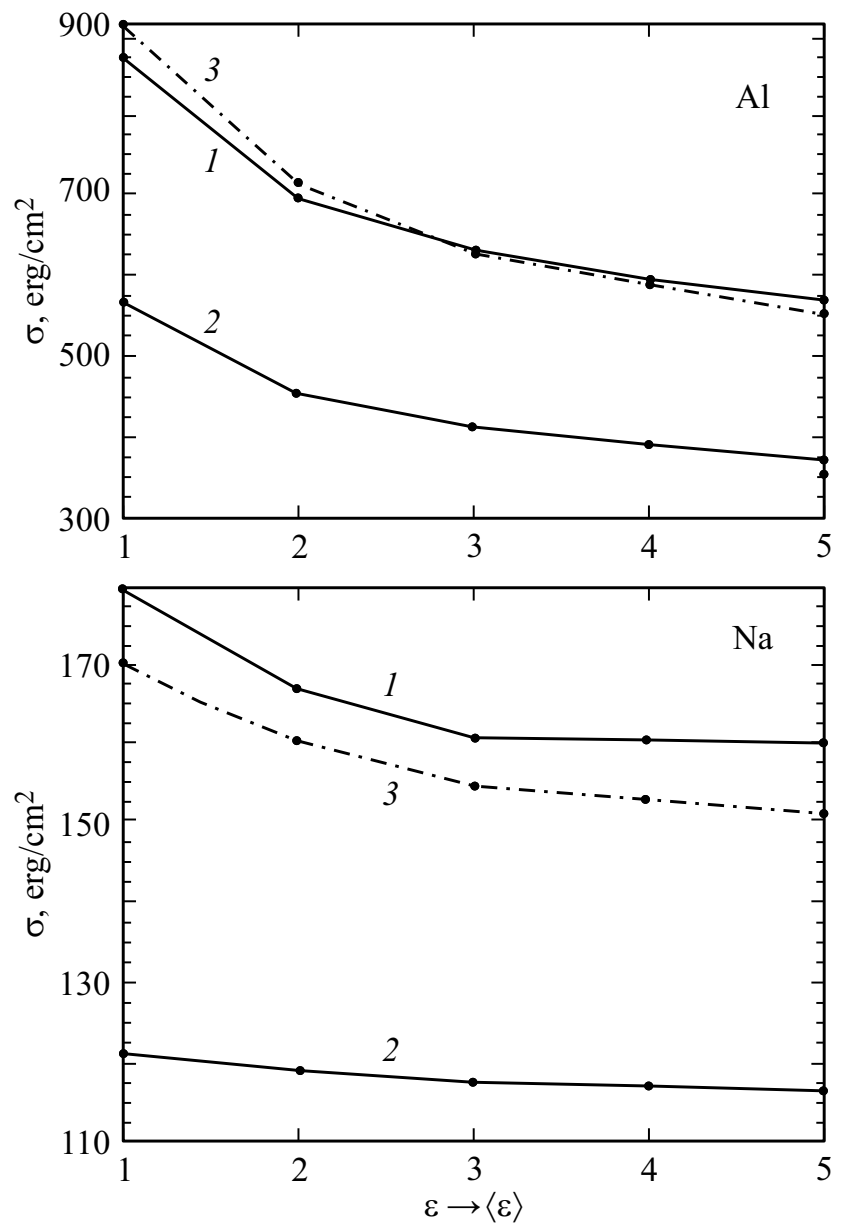

Рис. 4. Зависимость удельной поверхность энергии $\sigma(\varepsilon)$ для $\mathrm{Al}$ и $\mathrm{Na}\left(r_{s}=3.99 a_{0}\right)$, рассчитанная с функцией (21) для полного градиентного ряда (1) и только с поправкой Киржница (2). Для сравнения приведены значения, рассчитанные методом Кона-Шема (3). 

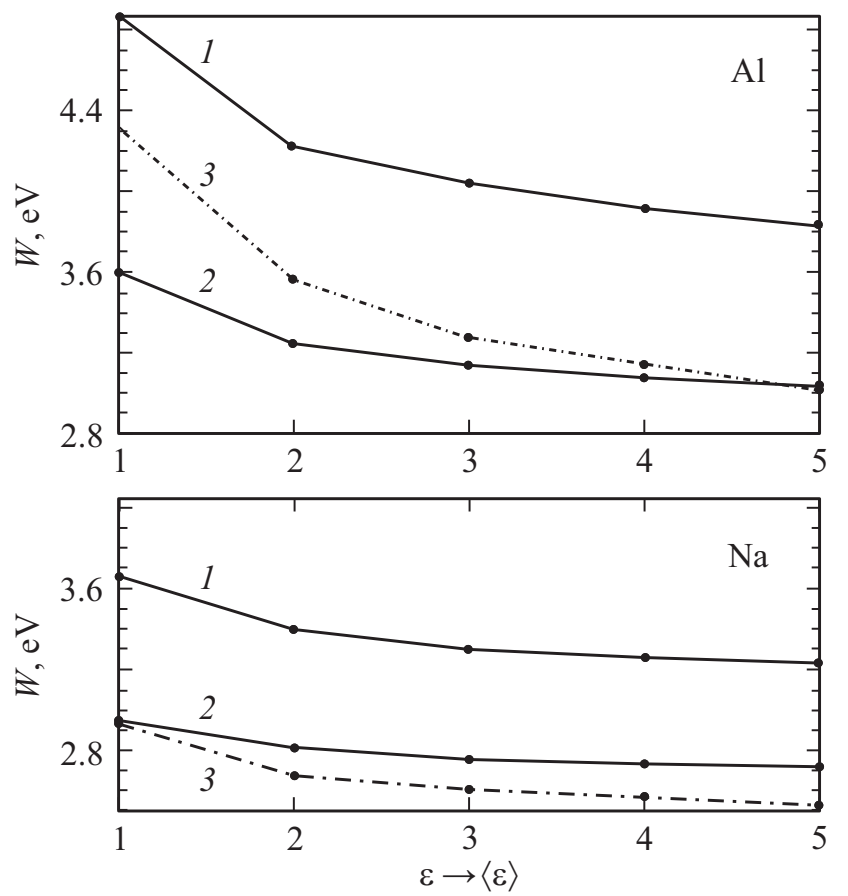

Рис. 5. Зависимость работы выхода электронов $W(\varepsilon)$ для $\mathrm{Al}$ и $\mathrm{Na}$, рассчитанная с функцией (21). Обозначения такие же как и на рис. 4.

„Вдавливанию“ профиля $\phi(z)$ в металл $[\phi(z)$ является компонентой $\left.v_{\text {eff }}(z)\right]$.

На рис. 4 и 5 приведены зависимости поверхностной энергии $\sigma(\varepsilon)$ и работы выхода электронов $W(\varepsilon)$. С увеличением константы $\varepsilon$ покрытия величины $\sigma$ и $W$ уменьшаются и выходят на плато. Расчетные значения наблюдаемой характеристики $W$, а также $\sigma$, слабо зависят от вида пробной функции (различие, в основном, наблюдается в третьей значащей цифре), но весьма чувствительны к градиентному приближению. Вычисления для полного градиентного ряда лучше согласуется с известными экспериментальными значениями при $\varepsilon=1$ и расчетом методом Кона-Шема для $\sigma$, но вместе с тем, дают завышенные значения для $W$. Расчеты только с градиентной поправкой Киржница $\left[C_{\mathrm{g} 2}, C_{\mathrm{g} 3}, C_{\mathrm{g} 4}=0\right.$ в (26)] приводят, наоборот, к лучшему согласию по $W$, чем по $\sigma$. При $\varepsilon=1$ экспериментальные величины $\sigma=926$ $\mathrm{erg} / \mathrm{cm}^{2}(\mathrm{Al}), 191 \mathrm{erg} / \mathrm{cm}^{2}(\mathrm{Na})$ и $W=4.25 \mathrm{eV}$ (Al), $2.35 \div 2.75 \mathrm{eV}(\mathrm{Na})$.

\section{4. Неоднородное покрытие поверхности}

Рассмотрим случай $\alpha=1 / 2$ на рис. 1 , когда центр металлического шара расположен на плоской границе двух диэлектриков. Ось $Z$ направлена перпендикулярно границе $\left(\theta_{m}=\pi / 2\right)$. „Сплющивая“ шар в диск (рис. $\left.6, a\right)$, мы получаем макроскопическую „пластину“ толщиной $L$ в диэлектрическом окружении - металл-диэлектрический сандвич с плоским интерфейсом (рис. 6, $b$ ).
Для сандвича выберем профиль электронного распределения в виде

$$
n(z)=\bar{n} \begin{cases}B_{1} e^{\left(z+Z_{1}\right) / \lambda_{1}}, & z<-Z_{1}, \\ 1-A_{1} e^{-\left(z+Z_{1}\right) / \lambda_{1}}, & -Z_{1} \leq z \leq 0, \\ 1-A_{2} e^{\left(z-Z_{2}\right) / \lambda_{2}}, & 0 \leq z \leq Z_{2}, \\ B_{2} e^{-\left(z-Z_{2}\right) / \lambda_{2}}, & z>Z_{2},\end{cases}
$$

где $Z_{1,2}=L / 2+\delta_{1,2}$.

Условие сшивки профиля (27) в $z=0$ для произвольного $L$ и условие электронейтральности подтверждают (12) и выполняются при $\delta_{1}=-\delta_{2}=0$. Последнее легко также проверить рассчитывая, например,

$$
\left[\frac{1}{2} \sigma_{\mathrm{t}}\left(\lambda_{1}, \delta_{1}\right)+\frac{1}{2} \sigma_{\mathrm{t}}\left(\lambda_{2}, \delta_{2}\right)\right]_{\lambda_{i} \equiv \lambda}=\sigma_{\mathrm{t}}(\lambda) .
$$

Ограничение условием $\delta_{i}=0$ является всего лишь следствием использования однопараметрической пробной функции.

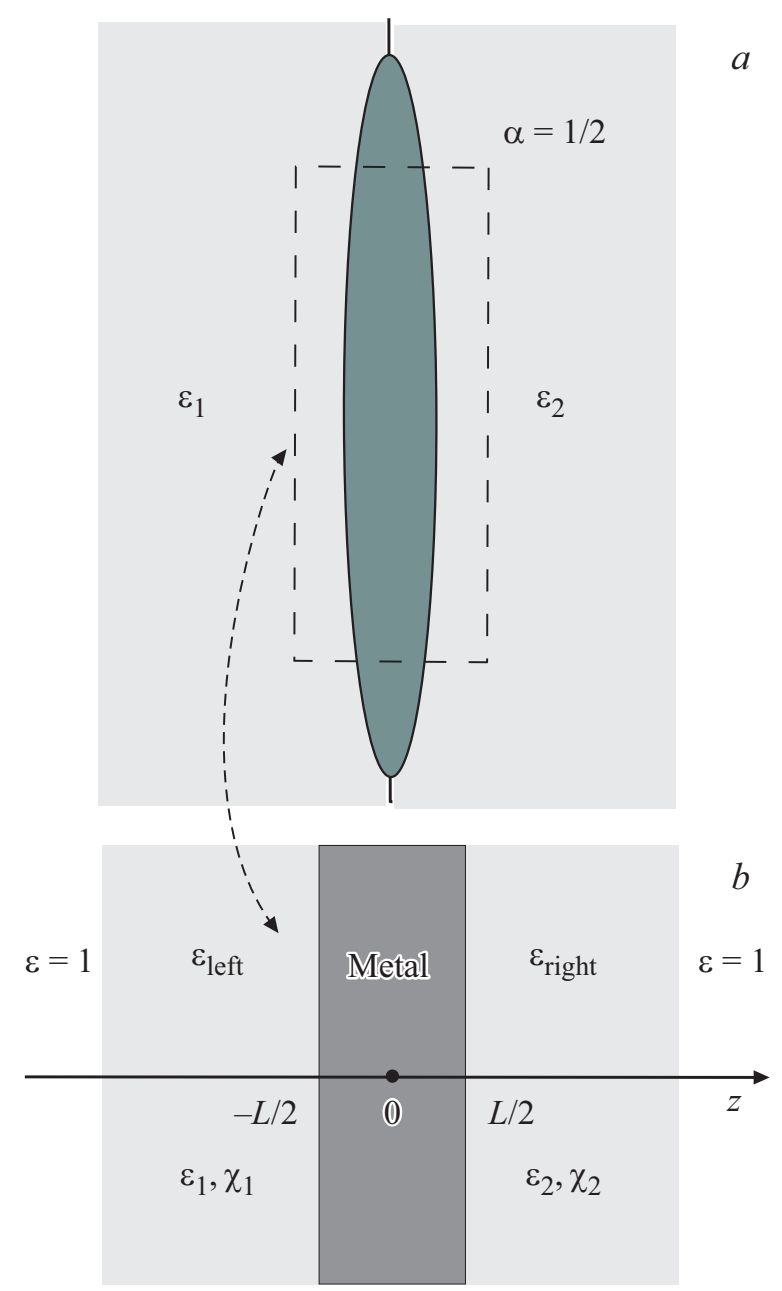

Рис. 6. Геометрическое построение, поясняющее связь связь частного случая $\alpha=1 / 2$ покрытия с металл-диэлетрическими наносандвичами из работы [6]. 
Подставляя (27) в выражение электростатического потенциала сандвича

$$
\phi(z)=-4 \pi \int_{z}^{\infty} d z^{\prime} \int_{z^{\prime}}^{\infty} d z^{\prime \prime} \frac{v\left(z^{\prime \prime}\right)}{\epsilon\left(z^{\prime \prime}\right)}, \quad \phi(\mp \infty)=0
$$

и сохраняя только экспоненциальную координатную зависимость при $L \rightarrow \infty$, получим

$$
\phi(z)=-2 \pi \bar{n} \lambda^{2} \begin{cases}\frac{1}{\langle\varepsilon\rangle} e^{(z+L / 2) / \lambda}, & z<-L / 2, \\ 1+\frac{1}{\langle\varepsilon\rangle}-e^{-(z+L / 2) / \lambda}, & -L / 2 \leq z \leq 0, \\ 1+\frac{1}{\langle\varepsilon\rangle}-e^{(z-L / 2) / \lambda}, & 0 \leq z \leq L / 2, \\ \frac{1}{\langle\varepsilon\rangle} e^{-(z-L / 2) / \lambda}, & z>L / 2,\end{cases}
$$

где

$$
\langle\varepsilon\rangle=\frac{\varepsilon_{1}+\varepsilon_{2}}{2} .
$$

Профиль (28) отражает эквипотенциальность поверхности пластины непосредственно на границе положительно заряженного фона. Поэтому $\phi(\mathbf{r})$ изменяется только по нормали к поверхности.

Таким образом для пластины можно применять то же выражение (26), используя (29), а для работы выхода $\bar{\phi}$ в (20) нужно заменить на значение потенциала в центре пластины $\phi(0)=-2 \pi \bar{n} \lambda^{2}\left(1+\langle\varepsilon\rangle^{-1}\right)$.

Формула (29) имеет аналогии. Во-первых, наблюдается прямая связь со значением электростатического потенциала на границе проводящего шара, симметрично расположенного между двумя диэлектриками с $\varepsilon_{1}$ и $\varepsilon_{2}$ [24]. Во-вторых, обнаруживается прямая связь формулы (29) с численными результатами метода КонаШема [6] поверхностной энергии и работы выхода электронов для металл-диэлектрических наносандвичей, например, нанопластины $\mathrm{Al}$, слева и справа от которой находятся диэлектрики: $\left\{\varepsilon_{\text {left }}\left|\mathrm{Al}_{L}\right| \varepsilon_{\text {right }}\right\}$ (рис. $6, b$ ).

В результатах работы [6] имели место особенности, которые не получили объяснения. Зависимости $W(L)$ для сандвичей $\left\{1\left|\mathrm{Al}_{L}\right| 5\right\}$ и $\left\{3\left|\mathrm{Al}_{L}\right| 3\right\}$ совпадали. Расчет для $\left\{1\left|\mathrm{Al}_{L}\right| 9\right\}$ и $\left\{5\left|\mathrm{Al}_{L}\right| 5\right\}$ дал тот же результат, т. е. работа выхода для асимметричных сандвичей $\left\{\varepsilon_{\text {left }}\left|\mathrm{Al}_{L}\right| \varepsilon_{\text {right }}\right\}$ совпадала с работой выхода симметричных сандвичей $\left\{\langle\varepsilon\rangle\left|\mathrm{Al}_{L}\right|\langle\varepsilon\rangle\right\}$ со среднеарифметическим значением $\langle\varepsilon\rangle=\left(\varepsilon_{\text {left }}+\varepsilon_{\text {right }}\right) / 2$ (см. рис. 5 в [6]). Для удельной поверхностной энергии, которая рассчитывалась среднеарифметически такого не наблюдалось. Профиль одноэлектронного эффективного потенциала представлял собой своеобразный „двуликий янус“" с горбом потенциала, например, для $\left\{1\left|\mathrm{Al}_{L}\right| 5\right\}$ со стороны вакуума $\left(\varepsilon_{\mathrm{left}}=1\right)$. Теперь на основании метода Ритца можно дать краткие комментарии.

Результаты вычислений методом Ритца для $\mathrm{Al}$ и $\mathrm{Na}$ с $\alpha=1 / 2$ приведены в табл. 2 и 3. Сопоставление значений $\sigma$ и $W$ при $\alpha=1 / 2 ; \varepsilon_{1}=3$ и $\varepsilon_{2}=1$, а также $\varepsilon_{1}=5$ и $\varepsilon_{2}=1$, показывают совпадение со значениями $\sigma$ и $W$ для $\langle\varepsilon\rangle=2$ и 3 на рис. 4 и 5.
Таблица 2. Результаты вычислений с функцией (21) для $\mathrm{Al}$ с

\begin{tabular}{|c|c|c|c|c|c|}
\hline$\alpha$ & $\varepsilon_{1}$ & $\varepsilon_{2}$ & $\lambda, a_{0}$ & $\sigma, \mathrm{erg} / \mathrm{cm}^{2}$ & $W, \mathrm{eV}$ \\
\hline $1 / 2$ & 1 & 1 & $\begin{array}{l}0.709 \\
0.602\end{array}$ & $\begin{array}{l}872 \\
567\end{array}$ & $\begin{array}{l}4.88 \\
3.60\end{array}$ \\
\hline $1 / 2$ & 2 & 1 & $\begin{array}{l}0.741 \\
0.638\end{array}$ & $\begin{array}{l}760 \\
497\end{array}$ & $\begin{array}{l}4.47 \\
3.39\end{array}$ \\
\hline $1 / 2$ & 3 & 1 & $\begin{array}{l}0.760 \\
0.661\end{array}$ & $\begin{array}{l}698 \\
456\end{array}$ & $\begin{array}{l}4.25 \\
3.27\end{array}$ \\
\hline $1 / 2$ & 4 & 1 & $\begin{array}{l}0.774 \\
0.678\end{array}$ & $\begin{array}{l}658 \\
430\end{array}$ & $\begin{array}{l}4.12 \\
3.20\end{array}$ \\
\hline $1 / 2$ & 5 & 1 & $\begin{array}{l}0.784 \\
0.687\end{array}$ & $\begin{array}{l}630 \\
411\end{array}$ & $\begin{array}{l}4.03 \\
3.16\end{array}$ \\
\hline $1 / 2$ & 4 & 3 & $\begin{array}{l}0.791 \\
0.695\end{array}$ & $\begin{array}{l}609 \\
397\end{array}$ & $\begin{array}{l}3.96 \\
3.12\end{array}$ \\
\hline $1 / 3$ & 2 & 1 & $\begin{array}{l}0.732 \\
0.628\end{array}$ & $\begin{array}{l}790 \\
516\end{array}$ & $\begin{array}{l}4.58 \\
3.44\end{array}$ \\
\hline $1 / 3$ & 3 & 1 & $\begin{array}{l}0.748 \\
0.647\end{array}$ & $\begin{array}{l}736 \\
481\end{array}$ & $\begin{array}{l}4.39 \\
3.34\end{array}$ \\
\hline $1 / 3$ & 4 & 1 & $\begin{array}{l}0.760 \\
0.661\end{array}$ & $\begin{array}{l}698 \\
456\end{array}$ & $\begin{array}{l}4.25 \\
3.27\end{array}$ \\
\hline $1 / 3$ & 5 & 1 & $\begin{array}{l}0.770 \\
0.671\end{array}$ & $\begin{array}{l}669 \\
438\end{array}$ & $\begin{array}{l}4.16 \\
3.22\end{array}$ \\
\hline $1 / 3$ & 4 & 3 & $\begin{array}{l}0.789 \\
0.692\end{array}$ & $\begin{array}{l}615 \\
402\end{array}$ & $\begin{array}{l}3.98 \\
3.13\end{array}$ \\
\hline $1 / 4$ & 2 & 1 & $\begin{array}{l}0.727 \\
0.623\end{array}$ & $\begin{array}{l}807 \\
527\end{array}$ & $\begin{array}{l}4.64 \\
3.48\end{array}$ \\
\hline $1 / 4$ & 3 & 1 & $\begin{array}{l}0.741 \\
0.638\end{array}$ & $\begin{array}{l}760 \\
497\end{array}$ & $\begin{array}{l}4.47 \\
3.39\end{array}$ \\
\hline $1 / 4$ & 4 & 1 & $\begin{array}{l}0.752 \\
0.651\end{array}$ & $\begin{array}{l}725 \\
474\end{array}$ & $\begin{array}{l}4.35 \\
3.32\end{array}$ \\
\hline $1 / 4$ & 4 & 3 & $\begin{array}{l}0.787 \\
0.691\end{array}$ & $\begin{array}{l}619 \\
404\end{array}$ & $\begin{array}{l}3.99 \\
3.14\end{array}$ \\
\hline
\end{tabular}
полным градиентным рядом (верхние значения) и с поправкой Киржница (нижние значения)

Появление горба потенциала для несимметричного сандвича $\left\{1\left|\mathrm{Al}_{L}\right| 5\right\}$ в [6] связано с перезарядкой поверхности. В соответствии с условием равновесия для химического потенциала $\mu(z)=$ const электроны перетекли с правой стороны пластины на левую. Метод Ритца с монотонными функциями не способен отобразить этот эффект.

В случае $\alpha \neq 1 / 2$ и $R \rightarrow \infty$ потенциал

$$
\phi(\mathbf{r})=\int d \mathbf{r}^{\prime} \frac{v\left(\mathbf{r}^{\prime}\right)}{\left|\mathbf{r}-\mathbf{r}^{\prime}\right| \epsilon\left(\mathbf{r}^{\prime}\right)}
$$

в аналитических функциях не выражается даже приближенно, вследствие того, что $\alpha$ не является малым параметром. В этом случае можно поступить следующим образом. 
Таблица 3. Результаты вычислений с функцией (21) для Na. Обозначения такие же как и в табл. 2

\begin{tabular}{|c|c|c|c|c|c|}
\hline$\alpha$ & $\varepsilon_{1}$ & $\varepsilon_{2}$ & $\lambda, a_{0}$ & $\sigma, \mathrm{erg} / \mathrm{cm}^{2}$ & $W, \mathrm{eV}$ \\
\hline $1 / 2$ & 1 & 1 & $\begin{array}{l}1.061 \\
0.749\end{array}$ & $\begin{array}{l}178 \\
121\end{array}$ & $\begin{array}{l}3.66 \\
2.94\end{array}$ \\
\hline $1 / 2$ & 2 & 1 & $\begin{array}{l}1.090 \\
0.775\end{array}$ & $\begin{array}{l}171 \\
119\end{array}$ & $\begin{array}{l}3.49 \\
2.86\end{array}$ \\
\hline $1 / 2$ & 3 & 1 & $\begin{array}{l}1.107 \\
0.789\end{array}$ & $\begin{array}{l}167 \\
118\end{array}$ & $\begin{array}{l}3.40 \\
2.82\end{array}$ \\
\hline $1 / 2$ & 4 & 1 & $\begin{array}{l}1.119 \\
0.799\end{array}$ & $\begin{array}{l}164 \\
117\end{array}$ & $\begin{array}{l}3.34 \\
2.79\end{array}$ \\
\hline $1 / 2$ & 5 & 1 & $\begin{array}{l}1.127 \\
0.805\end{array}$ & $\begin{array}{l}163 \\
116\end{array}$ & $\begin{array}{l}3.30 \\
2.77\end{array}$ \\
\hline $1 / 2$ & 4 & 3 & $\begin{array}{l}1.132 \\
0.810\end{array}$ & $\begin{array}{l}162 \\
116\end{array}$ & $\begin{array}{l}3.28 \\
2.76\end{array}$ \\
\hline $1 / 3$ & 2 & 1 & $\begin{array}{l}1.082 \\
0.768\end{array}$ & $\begin{array}{l}172 \\
120\end{array}$ & $\begin{array}{l}3.53 \\
2.88\end{array}$ \\
\hline $1 / 3$ & 3 & 1 & $\begin{array}{l}1.097 \\
0.780\end{array}$ & $\begin{array}{l}169 \\
118\end{array}$ & $\begin{array}{l}3.45 \\
2.84\end{array}$ \\
\hline $1 / 3$ & 4 & 1 & $\begin{array}{l}1.107 \\
0.789\end{array}$ & $\begin{array}{l}167 \\
118\end{array}$ & $\begin{array}{l}3.40 \\
2.82\end{array}$ \\
\hline $1 / 3$ & 5 & 1 & $\begin{array}{l}1.115 \\
0.796\end{array}$ & $\begin{array}{l}165 \\
117\end{array}$ & $\begin{array}{l}3.36 \\
2.80\end{array}$ \\
\hline $1 / 3$ & 4 & 3 & $\begin{array}{c}1.31 \\
0.809\end{array}$ & $\begin{array}{l}162 \\
116\end{array}$ & $\begin{array}{l}3.28 \\
2.76\end{array}$ \\
\hline $1 / 4$ & 2 & 1 & $\begin{array}{l}1.078 \\
0.764\end{array}$ & $\begin{array}{l}174 \\
120\end{array}$ & $\begin{array}{l}3.56 \\
2.89\end{array}$ \\
\hline $1 / 4$ & 3 & 1 & $\begin{array}{l}1.090 \\
0.775\end{array}$ & $\begin{array}{l}171 \\
119\end{array}$ & $\begin{array}{l}3.49 \\
2.86\end{array}$ \\
\hline $1 / 4$ & 4 & 1 & $\begin{array}{l}1.100 \\
0.783\end{array}$ & $\begin{array}{l}168 \\
118\end{array}$ & $\begin{array}{l}3.43 \\
2.84\end{array}$ \\
\hline $1 / 4$ & 4 & 3 & $\begin{array}{l}1.130 \\
0.808\end{array}$ & $\begin{array}{l}162 \\
116\end{array}$ & $\begin{array}{l}3.29 \\
2.76\end{array}$ \\
\hline
\end{tabular}

В электростатике при расчете емкости плоского конденсатора, частично заполненного разными диэлектриками, величины $\varepsilon_{i}$ входят в комбинации $\varepsilon_{i} S_{i}$, где $S_{i}=\alpha_{i} S-$ площади контактов. Можно предложить обобщение выражений (23) и (26) с помощью замены

$$
\widetilde{\varepsilon} \rightarrow \sum_{i} \varepsilon_{i} \alpha_{i}
$$

для произвольного числа контактов плоской металлической поверхности с диэлектриками. В рассматриваемом случае двух диэлектриков

$$
\widetilde{\varepsilon} \rightarrow \varepsilon_{1} \alpha+\varepsilon_{2}(1-\alpha)
$$

и для $\alpha=1 / 2$ имеем $\widetilde{\varepsilon}=\langle\varepsilon\rangle$.
Значения параметра $\lambda$, величины $\sigma$ и $W$ при $\varepsilon_{1}=\varepsilon_{2}$ соответствуют однородным покрытиям и точкам на рис. 2 , 4 и 5. Случай $\varepsilon_{1}=\varepsilon_{2}=1$ соответствуют интерфейсу металл-вакуум и „первым“ точкам на тех же рисунках.

Неоднородность покрытия формально определяется слагаемыми

$$
\bar{n}^{2} \lambda^{3} C_{\mathrm{q}}\left(1+\frac{1}{\varepsilon_{1} \alpha+\varepsilon_{2}(1-\alpha)}\right)
$$

и

$$
\bar{\phi}=-2 \pi \bar{n} \lambda^{2}\left(1+\frac{1}{\varepsilon_{1} \alpha+\varepsilon_{2}(1-\alpha)}\right)
$$

для $\sigma$ и $W$ в (26) и (20), соответственно. Поэтому, если для различных значений $\alpha, \varepsilon_{1}, \varepsilon_{2}$ комбинация $\varepsilon_{1} \alpha+\varepsilon_{2}(1-\alpha)$ является целочисленной, то точки на рис. 4 и 5 для однородных покрытий соответствуют именно этим числам.

Таким образом рис. 4 и 5 будут ключевыми а, используя соотношение (29) в качестве масштабного преобразования, можно оценить значения $\sigma$ и $W$ для неоднородного покрытия.

Комментируя роль параметров $\delta_{1}$ и $\delta_{2}$, следует указать, что выбирая монотонные пробные функции вида (21) или (22), условие электронейтральности в плоском случае дает возможность использовать только один вариационный параметр $(\lambda)$. Поэтому роль $\delta_{1}$ и $\delta_{2}$ в данном подходе сведена к выводу условия равенства (12). Точно задача для сандвича находится решением системы одномерных уравнений Кона-Шема и Пуассона [6]. В трехмерном случае эти решения должны удовлетворять условию

$$
\mu\left(\varepsilon_{i}, x, y, z\right)=\text { const }=-W\left(\varepsilon_{i}\right) .
$$

Вычислительная процедура в такой постановке до сих пор не реализована.

Таким образом в работе сделана попытка аналитически показать, что работа выхода электронов $W(\widetilde{\varepsilon})$, как энергия электрона в глубине металла, отсчитанная от вакуумного уровня, является изотропной, независимо от формы поверхности металлического образца и диэлектрического покрытия. Такой вывод следует из эквипотенциальности металлической поверхности [10-12].

Барьер Шоттки для неоднородного покрытия

$$
\Phi^{(i)}=W(\widetilde{\varepsilon})-\chi^{(i)},
$$

наоборот, является анизотропной величиной и в процесcax переноса определяется наибольшей величиной $\chi^{(i)}$ (наименьшим $\left.\Phi^{(i)}\right) \cdot \chi=0.1,0.2,0.45,0.68,1.1,1.35,4.05$ и $4 \mathrm{eV}$ для $\mathrm{Ne}, \mathrm{Ar}, \mathrm{Kr}, \mathrm{Xe}, \mathrm{Al}_{2} \mathrm{O}_{3}, \mathrm{Si}$ и $\mathrm{Ge}$, соответственно.

\section{5. Заключение}

В данной работе в рамках метода Ритца исследованы поверхностная энергия и работы выхода электронов металлической плоской поверхности с неоднородным 
диэлектрическим покрытием. Для однородного покрытия расчетные величины нечувствительны к выбору однопараметрических функций для электронного профиля, но чувствительны к градиентному ряду кинетической энергии невзаимодействующих электронов. Тем не менее это не повлияло на качественные выводы работы. Проведено также сравнение результатов вычислений методов Ритца и Кона-Шема.

Анализ в рамках метода Ритца позволил дать интерпретацию некоторых особенностей расчетных результатов метода Кона-Шема для ассиметричных металлдиэлектрических наносандвичей.

Предложено влияние неоднородности покрытия на характеристики металлической поверхности свести масштабированием к случаю однородного покрытия.

Показано, что работа выхода электронов, отсчитанная от вакуумного уровня, является скалярной величиной, независимо от диэлектрического покрытия. Барьер Шоттки для композитного покрытия, наоборот, является величиной анизотропной.

Обсуждая возможность наблюдения зависимостей $W(\varepsilon, \chi)$ для различных контактов, следует рассмотреть два режима ситуаций. Если работа выхода электронов в вакуум $W(\varepsilon, \chi)$ измеряется в результате внешнего фотоэффекта, то такая возможность относится только к металлу с диэлектрическим покрытием толщиной меньше длины свободного пробега (отсутствие энергообмена электрона с изолятором). В случае энергообмена величина $W$ может быть восстановлена по формуле (32) и измерению $\Phi$ в результате внутреннего фотоэффекта. В приведенных ситуациях в результате решения обратной задачи по измерениям $W(\varepsilon, \chi)$ можно оценить длину свободного пробега электронов в изоляторе.

В нашем исследовании мы предполагали отсутствие химических изменений в контакте, что достаточно правдоподобно для изоляторов с малым $\chi$. Для полупроводников $\mathrm{Si}, \mathrm{Ge}$ наличие поверхностных состояний приводит к пиннингу энергии Ферми. В этом случае задача решается кластерным методом (см., например, [26]).

В методе Ритца для металлической поверхности с диэлектрическим покрытием удельная поверхностная энергия выполняет вспомогательную роль: в результате оптимизации определялось „истинное“ значение параметра $\lambda\left(\varepsilon_{1}, \varepsilon_{2}\right)$, которое необходимо для измеряемой характеристики-работы выхода электронов $W(\lambda)$. Можно предложить следующее практическое использование полученных результатов для $\sigma\left(\varepsilon_{1}, \varepsilon_{2}\right)$.

Экспериментально поверхностное натяжение определяется краевым углом смачивания по закону Юнга [27]. При этом полученная в данной работе зависимость $\left.\sigma\left(\alpha, \varepsilon_{1}, \varepsilon_{2}\right)\right|_{\varepsilon_{2}=1}$ в определенном смысле моделирует металлическую каплю, касающуюся площадью $\alpha S$ диэлектрической подложки с константой $\varepsilon_{1}$. Предложенный в данной работе подход, возможно, позволит уточнить измерения $\sigma$.

\section{Благодарности}

Автор выражает благодарность А.В. Бабичу за помощь в проведении вычислений.

\section{Конфликт интересов}

Автор заявляет об отсутствии конфликта интересов

\section{Список литературы}

[1] E.G. Barbagiovanni, D.J. Lockwood, P.J. Simpson, L.V. Goncharova. J. Appl. Phys. 111, 034307 (2012).

[2] M. Liu, Y. Han, L. Tang, J.-F. Jia, Q.-K. Xue, F. Liu. Phys. Rev. B 86, 125427 (2012).

[3] R.Y. Liu, A. Huang, C.C. Huang, C.-Y. Lee, C.-H. Lin, C.-M. Cheng, K.-D. Tsuei, H.-T. Jeng, I. Matsuda, S.-J. Tang. Phys. Rev. B 92, 115415 (2015).

[4] P.M. Dinh, P.-G. Reinhard, E. Suraud. Phys. Rep. 485, 43 (2010).

[5] В.В. Погосов, А.В. Бабич, П.В. Вакула, А.Г. Кравцова. ЖТФ 81, 11, 150 (2011).

[6] А.В. Бабич, В.В. Погосов. ФТТ 55, 177 (2013).

[7] В.В. Погосов, А.В. Бабич, П.В. Вакула. ФТТ 55, 2004 (2013).

[8] S. Prada, U. Martinez, G. Pacchioni. Phys. Rev. B 78, 235423 (2008).

[9] R.T. Tung. Appl. Phys. Rev. 1, 011304 (2014).

[10] N.W. Ashcroft, N.D. Mermin. Solid State Physics. Holt, Rinehart and Winston, N.Y. (1976). Part 18 (Fig. 18.3).

[11] C.J. Fall. Ph.D. Dissertation. École Polytechnique Fédérale de Lausanne (1999).

[12] V.V. Pogosov, V.P. Kurbatsky. ЖЭТФ 119, 350 (2001).

[13] C.J. Fall, N. Binggeli, A. Baldereschi. Phys. Rev. Lett. 88, 156802 (2002).

[14] A.V. Babich, V.V. Pogosov. Surf. Sci. 603, 2393 (2009).

[15] L. Gao, J. Souto-Casares, J.R. Chelikowsky, A.A. Demkov. J. Chem. Phys. 147, 214301 (2017).

[16] В.А. Тиньков. Успехи физ. мет. 7, 117 (2006).

[17] I.T. Iakubov, A.G. Khrapak, L.I. Podlubny, V.V. Pogosov. Solid State Commun. 53, 427 (1985).

[18] R. Smoluchowski. Phys. Rev. 60, 661 (1941).

[19] J.R. Smith. Phys. Rev. 181, 522 (1969).

[20] C.H. Hodges. Can. J. Phys. 51, 1428 (1973).

[21] J.P. Perdew, H.Q. Tran, E.D. Smith. Phys. Rev. B 42, 11627 (1990).

[22] В.В. Погосов. ФТТ 61, 224 (2019).

[23] K. Hirabayashi. Phys. Rev. B 3, 4023 (1971).

[24] В.В. Батыгин, И.Н. Топтыгин. Сб. задач по элетродинамике и специальной теории относительности. Лань, С.-Петербург-Москва-Краснодар (2010). 480 с.

[25] V.V. Pogosov. Chem. Phys. Lett. 193473 (1992).

[26] V.G. Zavodinsky, I.A. Kuyanov. Superlattice. Microst., 24, 55 (1998).

[27] Э.Э. Шпильрайн, К.А. Якимович, Е.Е. Тоцкий, Д.Л. Тимпорт, В.А. Фомин Теплофизические свойства щелочных металлов. Изд-во стандартов, М. (1970). 487 с.

Редактор Т.Н. Василевская 